\section{Kidney Blood Pressure Research}

\title{
Gestational Estimated Glomerular Filtration Rate and Adverse Maternofetal Outcomes
}

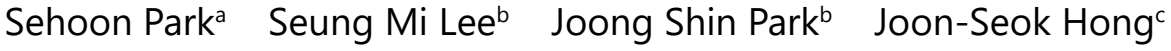 \\ Ho Jun Chin ${ }^{d, e} \quad K i$ Young Nad,e Dong Ki Kim ${ }^{e, f} \quad$ Kook-Hwan Oh ${ }^{f}$ \\ Kwon Wook Joo ${ }^{e, f}$ Yon Su Kim ${ }^{a, e, f}$ Hajeong Lee ${ }^{e, f}$ \\ aDepartment of Biomedical Sciences, Seoul National University College of Medicine, Seoul, \\ 'Department of Obstetrics and Gynecology, Seoul National University College of Medicine, Seoul, \\ 'Department of Obstetrics and Gynecology, Seoul National University Bundang Hospital, Seongnam, \\ Gyeonggi-do, 'Department of Internal Medicine, Seoul National University Bundang Hospital, \\ Seongnam, Gyeonggi-do, eKidney Research Institute, Seoul National University College of Medicine, \\ Seoul, 'Department of Internal Medicine, Seoul National University Hospital, Seoul, Korea
}

\section{Key Words}

Pregnancy - Glomerular filtration rate - Preterm - Preeclampsia - Low birth weight • Hyperfiltration $•$ Chronic kidney disease

\begin{abstract}
Background/Aims: The association between gestational estimated glomerular filtration rate (eGFR) and adverse pregnancy outcomes has not been fully investigated. Methods: This observational cohort study included pregnancy cases of singleton mothers whose serum creatinine levels were measured during pregnancy at two tertiary hospitals in Korea from 2000 to 2015. Those with identified substantial renal function impairment (eGFR $<60 \mathrm{~mL} / \mathrm{min} / 1.73$ $\mathrm{m}^{2}$ at baseline, during, or after pregnancy) were excluded. The Chronic Kidney Disease Epidemiology Collaboration equation was used for the eGFR calculation. We computed the time-averaged eGFR during gestation to determine representative values when there were multiple measurements. We studied the following three gestational complications: preterm birth ( $<37$ weeks' gestational age), low birth weight $(<2.5 \mathrm{~kg})$, and preeclampsia. Results: Among the 12,899 studied pregnancies, 4,360 cases experienced one or more gestational complications. The adjusted odds ratio (aOR) and 95\% confidence interval of composite gestational complications for eGFR ranges other than the reference range of $120-150 \mathrm{~mL} /$ $\mathrm{min} / 1.73 \mathrm{~m}^{2}$ were: $\geq 150 \mathrm{~mL} / \mathrm{min} / 1.73 \mathrm{~m}^{2}$, aOR 1.64 (1.38-1.95), $\mathrm{P}<0.001 ; 90-120 \mathrm{~mL} / \mathrm{min} / 1.73 \mathrm{~m}^{2}$, aOR 1.41 (1.28-1.56), $\mathrm{P}<0.001$; and $60-90 \mathrm{~mL} / \mathrm{min} / 1.73 \mathrm{~m}^{2}$, aOR 2.56 (1.70-3.84), $\mathrm{P}<0.001$. Incidence of preterm birth or low birth weight showed similar U-shaped association with eGFR values; otherwise, preeclampsia or small for gestational age occurred more often in mothers with a lower gestational eGFR than in those with a higher value. Conclusion: Considering the
\end{abstract}




\section{Kidney Blood Pressure Research}

Kidney Blood Press Res 2018;43:1688-1698
\begin{tabular}{l|l} 
DOI: 10.1159/000494746 & (c) 2018 The Author(s). Published by S. Karger AG, Basel \\
Published online: 31 October 2018 & ww.karger.com/kbr
\end{tabular}

Park et al.: Pregnancy Complications and Gestational eGFR

unique association between gestational eGFR and pregnancy outcomes, carefully interpreting these results may help predict obstetric complications.

\section{Introduction}

A substantial hemodynamic adaptation process occurs during pregnancy, which is important for safe gestation $[1,2]$. When this process is impaired, the risk of obstetric complications increases, and several biomarkers may reflect inappropriate hemodynamic alteration [3-6]. Considering that the underlying kidney dysfunction is significantly related to a higher incidence of adverse pregnancy outcomes, renal function might play a key role in the mother's hemodynamic adaptation to pregnancy [7]. Therefore, information on the underlying kidney function is valuable for stratifying the mothers' risks. However, the baseline renal status is often not evaluated before pregnancy, although there is a nonnegligible prevalence of early chronic kidney disease (CKD) in childbearing-aged women [8]. In addition, assessment of renal function during pregnancy has not been suggested in current guidelines for antenatal care, as the clinical significance of measured kidney function parameter has yet been well demonstrated in a large cohort $[9,10]$.

Previously, to evaluate renal parameters during pregnancy, we focused on midterm hyperfiltration and investigated the estimated glomerular filtration rate (eGFR) in the second trimester and its association with pregnancy outcomes [11]. We demonstrated that a prominent non-linear, U-shaped association exists between midterm eGFR and gestational complications, and the optimal midterm eGFR range was $120-150 \mathrm{~mL} / \mathrm{min} / 1.73 \mathrm{~m}^{2}$ using the Chronic Kidney Disease Epidemiology Collaboration (CKD-EPI) method [12]. However, the serum creatinine $(\mathrm{sCr}$ ) level was measured in only a small portion of mothers during their second trimester; therefore, selection bias was possible.

In this study, we further expanded our previous idea by screening all mothers whose sCr/eGFR was measured during pregnancy. We evaluated the association between eGFR during the gestation period and adverse pregnancy outcomes, using the time-averaged eGFR (TA-eGFR) level to integrate multiple values that were measured during pregnancy.

\section{Materials and Methods}

\section{Ethics statement}

The study was approved by the institutional review boards of Seoul National University Hospital and Bundang Seoul National University Hospital (H-1604-028-753) and performed according to the principles of the Declaration of Helsinki. Informed consent was waived because this was an observational cohort study without medical interventions.

\section{Study population}

We screened all pregnancy records of singleton mothers who delivered between 2000 and 2015 and whose sCr levels were measured during pregnancy. Additionally, we did not include pregnancy cases of mothers with substantially impaired renal function (i.e., when any measured eGFR less than $60 \mathrm{~mL} /$ $\min / 1.73 \mathrm{~m}^{2}$ at baseline, during, or after pregnancy was identified). Furthermore, pregnancies with unknown estimated conception date (subtracting the born child's gestational age from the delivery date) were excluded, as we could not confirm the pregnancy period.

\section{Data collection}

We collected data on clinical characteristics by retrospectively reviewing the participants' electronic health records. We collected data on age, height, baseline weight and weight change until delivery, body mass index (BMI), and history of hypertension or diabetes mellitus. We also collected data on the following 


\section{Kidney Blood Pressure Research}

Park et al.: Pregnancy Complications and Gestational eGFR

pregnancy characteristics: history of full-term and preterm birth, miscarriage/still birth history, the born child's birth weight and gestational age, and delivery method (cesarean or vaginal).

All sCr values that were included in the study were measured at each hospital's laboratory facilities (Roche Diagnostics) and recalibrated into an isotope-dilution mass spectrometry assay value. We used the CKD-EPI equation to calculate the eGFR values, which was also validated in the nation [12, 13]. The participants' blood pressure levels were measured upon admission for delivery, and dipstick urine albumin test results were collected before and during pregnancy.

To determine the underlying kidney function, we defined the baseline kidney function period as between 3 years from the delivery date and before the estimated conception date. The baseline kidney function was stratified among mothers with both eGFR and albuminuria test results that were available at the baseline period. Because we included pregnancies of mothers with eGFR greater than or equal to 60 $\mathrm{mL} / \mathrm{min} / 1.73 \mathrm{~m}^{2}$, those without baseline albuminuria results were classified as mothers without evidence of underlying CKD, and others were considered to have baseline CKD [14].

\section{Outcome measurement}

We collected data on preterm birth events, low birth weight, small for gestational age (SGA), and preeclampsia as adverse pregnancy outcomes, and the composite outcome was also analyzed. We followed the definition of the International Classification of Diseases, 10th Revision (ICD-10). Preterm birth was defined as gestational age less than 37 weeks at delivery, and we further stratified the birth periods as moderate-tolate preterm birth (gestational age $\geq 32$ weeks and $<37$ weeks) and very preterm birth (gestational age $\geq 28$ weeks and $<32$ weeks) $[15,16]$. Low birth weight and very low birth weight were defined as the born child's birth weight of less than $2.5 \mathrm{~kg}$ and less than $1.5 \mathrm{~kg}$, respectively. SGA was classified as a newborn with birth weight below the 10th percentile of sex-specific birth weight for gestational age. Preeclampsia events were confirmed based on the attending obstetricians' diagnoses.

\section{Statistical analysis}

We reported categorical variables as frequencies (percentages) and used chi-squared test to evaluate differences between subgroups. We reported continuous variables as the median (interquartile range) and used Kruskal-Wallis's test to assess between-group differences, because the continuous variables were not normally distributed according to Shapiro-Wilk's normality test.

We plotted the association between TA-eGFR and risk of gestational complications with generalized additive models, using the outcome as a binomial variable and eGFR as the explanatory variable. Univariable and multivariable logistic regression model analyses were performed using the subgroup with the lowest incidence of each adverse pregnancy outcome as the reference. In our multivariable analysis, the following variables were adjusted: age, baseline BMI, weight gain during pregnancy, multiparity, preterm birth history, hypertension or diabetes mellitus history, and presence of hypertension during pregnancy or gestational diabetes mellitus. Hypertension during pregnancy was not adjusted for preeclampsia outcomes because preeclampsia itself is a gestational hypertensive disorder. Multivariate imputation using a classification and regression tree method was performed using the "mice" package in R to impute missing data for the baseline BMI $(3,146$ cases $)$ and weight gain intra-gestation $(2,594$ cases).

We performed a multivariable receiver operating characteristic (ROC) curve analysis. We tested whether the area under the curve (AUC) significantly increased when we added a risk score that was calculated by eGFR ranges, using the "pROC" package in R. The risk scores were considered to increase one point from the lowest risk subgroup of each gestational complication to the high-risk subgroups. Other variables that were included in the ROC curve analysis were age; baseline BMI and weight gain during gestation; multiparity; history of preterm birth, hypertension, or diabetes mellitus; and gestational hypertension and diabetes mellitus. Similarly as above, gestational hypertension was excluded from the analysis of preeclampsia.

All statistical analyses were performed using $\mathrm{R}$ (version 3.4.0, the R foundation), and a two-sided P-value $<0.05$ indicated statistical significance. 


\section{Kidney Bloód Pressure Research}

Park et al.: Pregnancy Complications and Gestational eGFR

\section{Results}

\section{Study population}

There were 13,552 singleton-delivery cases of women whose sCr levels were measured during pregnancy (Fig. 1). Those whose estimated conception date was unavailable $(n=567)$ were excluded. We additionally excluded pregnancies of women with substantially impaired renal function $(\mathrm{n}=86)$, as they included pregnancies during dialysis or severe AKI events and showed extreme incidences of gestational complications [70/86 (81.4\%) composite outcomes, 66/86 (76.7\%) preterm births, 36/86 (41.9\%) preeclampsia, 29/45 (64.4\%) low birth weights and 14/45 (31.1\%) SGA events]. After the exclusion 12, 899 pregnancies were included in the final cohort. The $\mathrm{sCr} /$ eGFR was available for most pregnancies $(n=11,007)$ in the third trimester, and it was available in some during their first $(n=1,448)$ or second $(n=2,537)$ trimester. When stratified according to TA-eGFR during gestation, 124, 2,795, 9,341, and 639 cases had gestational TA-eGFR ranges of $60-90,90-120,120-150$, and $>150 \mathrm{~mL} /$ $\min / 1.73 \mathrm{~m}^{2}$, respectively.

\section{Clinical and pregnancy characteristics}

We identified several differences in the characteristics between the study subgroups (Table 1). Those who had a TAeGFR level $\geq 150 \mathrm{~mL} / \mathrm{min} / 1.73 \mathrm{~m}^{2}$ were the youngest, and they had the lowest baseline $\mathrm{BMI}$ and weight gain during pregnancy. They were more likely to be primiparous and experienced fewer obstetric complications than other groups. Preexisting hypertension

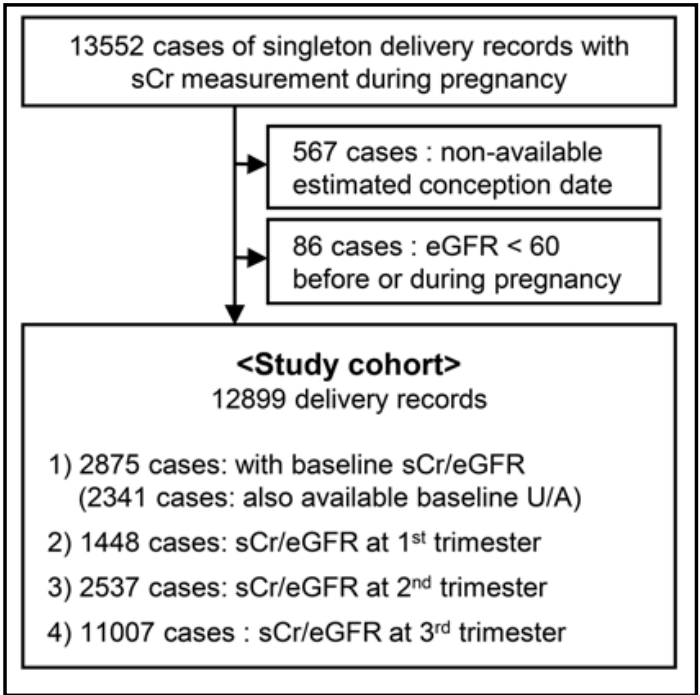

Fig. 1. Flow diagram of the study population. eGFR: estimated glomerular filtration rate; sCr: serum creatinine level; U/A, urinalysis.

Table 1. Baseline, pregnancy characteristics, and calculated eGFR values according to time-averaged eGFR during gestation. $\mathrm{SCr}$, serum creatinine, eGFR, estimated glomerular filtration rate

\begin{tabular}{|c|c|c|c|c|c|}
\hline Characteristics & $\begin{array}{c}60 \leq \mathrm{eGFR}<90 \\
(\mathrm{n}=124)\end{array}$ & $\begin{array}{c}90 \leq \text { eGFR }<120 \\
(\mathrm{n}=2795)\end{array}$ & $\begin{array}{c}120 \leq \text { eGFR }<150 \\
(\mathrm{n}=9341)\end{array}$ & $\begin{array}{l}\text { eGFR } \geq 150 \\
(n=639)\end{array}$ & $P$ value \\
\hline Age (years) & $33(30-36)$ & $35(33-38)$ & $32(30-35)$ & $31(28-34)$ & $<0.001$ \\
\hline Body mass index $\left(\mathrm{kg} / \mathrm{m}^{2}\right)$ & $21.2(19.6-23.1)$ & $21.2(19.6-23.4)$ & $20.9(19.3-22.9)$ & 20.7 (19.1-22.7) & $<0.001$ \\
\hline Multiparity (n(\%)) & $52(41.6)$ & $1579(56.5)$ & $5072(54.3)$ & $300(46.9)$ & $<0.001$ \\
\hline Presence of preterm birth history (n(\%)) & $7(5.6)$ & $241(8.6)$ & $674(7.2)$ & $34(5.3)$ & 0.01 \\
\hline Presence of stillbirth/miscarriage history (n(\%)) & $46(37.1)$ & $1094(39.1)$ & $2999(32.1)$ & $117(18.3)$ & $<0.001$ \\
\hline Previous history of hypertension (n(\%)) & $47(37.9)$ & $280(10.0)$ & $586(6.3)$ & $35(5.5)$ & $<0.001$ \\
\hline Previous history of diabetes mellitus (n(\%)) & $12(9.7)$ & $280(10.0)$ & $586(6.3)$ & $35(5.5)$ & $<0.001$ \\
\hline Weight gain during pregnancy $(\mathrm{kg})$ & $13.0(10.1-18.9)$ & $13.0(9.8-16.2)$ & $12.8(9.7-16.0)$ & $12.0(8.3-15.0)$ & $<0.001$ \\
\hline Hypertension during pregnancy (n(\%)) & $37(29.8)$ & $249(8.9)$ & $394(4.2)$ & $25(3.9)$ & $<0.001$ \\
\hline aSystolic blood pressure $(\mathrm{mmHg})$ & $137(124-152)$ & $121(111-134)$ & $119(110-129)$ & $119(110-130)$ & \\
\hline aDiastolic blood pressure (mmHg) & $84(71-97)$ & $75(68-86)$ & $71(66-80)$ & $70(62-78)$ & \\
\hline Gestational diabetes mellitus (n(\%)) & $11(8.9)$ & $194(6.9)$ & $442(4.7)$ & $32(5.0)$ & 0.001 \\
\hline bUrine albumin (dipstick) & & & & & $<0.001$ \\
\hline$(-)$ & $54(50.5)$ & $1766(73.1)$ & $6993(84.4)$ & $473(86.5)$ & \\
\hline $1^{+}$ & $6(5.6)$ & 309 (12.8) & $783(9.4)$ & $45(8.2)$ & \\
\hline $2^{+}$ & $18(16.8)$ & $165(6.8)$ & $315(3.8)$ & $16(2.9)$ & \\
\hline$>2^{+}$ & $29(27.1)$ & $175(7.2)$ & $198(2.4)$ & $13(2.4)$ & \\
\hline Cesarean section (n(\%)) & $54(43.2)$ & $1460(52.2)$ & $4264(45.6)$ & $243(38.0)$ & $<0.001$ \\
\hline \multicolumn{6}{|l|}{$\mathrm{eGFR}\left(\mathrm{mL} / \mathrm{min} / 1.73 \mathrm{~m}^{2}\right)$} \\
\hline cBaseline $(n=2875)$ & $107.1(95.2-118.9)$ & $114.3(104.8-120.3)$ & $121.4(115.9-128.4)$ & $127.5(120.5-137.6)$ & $<0.001$ \\
\hline \multicolumn{6}{|l|}{ dDuring gestation } \\
\hline First trimester $(n=1448)$ & $85.8(85.3-105.5)$ & $116.3(111.0-119.8)$ & $127.4(121.7-133.8)$ & $153.9(138.5-163.2)$ & $<0.001$ \\
\hline Second trimester $(n=2537)$ & $86.9(77.9-96.1)$ & $118.0(114.6-122.2)$ & $132.6(126.6-140.7)$ & $162.0(155.7-186.2)$ & $<0.001$ \\
\hline Third trimester $(\mathrm{n}=11007)$ & 83.5 (80.2-87.5) & $116.4(112.8-118.9)$ & $129.9(124.9-135.4)$ & $163.2(158.7-178.6)$ & $<0.001$ \\
\hline The number of sCr/eGFR measurements & & & & & $<0.001$ \\
\hline 1 & $77(62.1)$ & $1861(66.6)$ & $6591(70.6)$ & $448(76.4)$ & \\
\hline 2 & $17(13.7)$ & $488(17.5)$ & $1622(17.4)$ & $99(15.5)$ & \\
\hline$\geq 3$ & $30(24.2)$ & $446(16.0)$ & $1128(12.1)$ & $52(8.1)$ & \\
\hline
\end{tabular}




\section{Kidney Blood Pressure Research}

Table 2. Pregnancy outcomes according to time-averaged eGFR values during gestation. eGFR, estimated glomerular filtration rate

\begin{tabular}{|c|c|c|c|c|c|}
\hline Outcomes & $\begin{array}{c}60 \leq \mathrm{eGFR}<90 \\
(\mathrm{n}=124)\end{array}$ & $\begin{array}{c}90 \leq \mathrm{eGFR}<120 \\
(\mathrm{n}=2795)\end{array}$ & $\begin{array}{c}120 \leq \mathrm{eGFR}<150 \\
(\mathrm{n}=9341)\end{array}$ & $\begin{array}{c}\text { eGFR } \geq 150 \\
(n=639)\end{array}$ & $P$ value \\
\hline Composite outcome & $73(58.9)$ & $1060(37.9)$ & $2940(31.5)$ & $287(44.9)$ & $<0.001$ \\
\hline Preterm birth $(<37$ weeks) $(\mathrm{n}(\%))$ & $59(47.6)$ & $768(27.5)$ & $2321(24.8)$ & $259(40.5)$ & $<0.001$ \\
\hline Moderate to late preterm ( 32 to $<37$ weeks) & $39(31.5)$ & $498(17.8)$ & $1530(16.4)$ & $151(23.6)$ & $<0.001$ \\
\hline Very preterm $(28$ to $<32$ weeks $)(\mathrm{n}(\%))$ & $14(11.3)$ & $130(4.7)$ & $364(3.9)$ & $46(7.2)$ & $<0.001$ \\
\hline Extremely preterm $(<28$ weeks) $(\mathrm{n}(\%))$ & $6(4.8)$ & $140(5.0)$ & $427(4.6)$ & $62(9.7)$ & $<0.001$ \\
\hline Gestational age (weeks) & $37.0(34-39)$ & $38(36-39)$ & $38(37-39)$ & $37(34-39)$ & $<0.001$ \\
\hline Low birth weight $(<2.5 \mathrm{~kg})(\mathrm{n}(\%))$ & $50(40.3)$ & $607(21.7)$ & $1567(16.8)$ & $173(27.1)$ & $<0.001$ \\
\hline Very low birth weight $(<1.5 \mathrm{~kg})(\mathrm{n}(\%))$ & $18(14.5)$ & $220(7.9)$ & $493(5.3)$ & $57(8.9)$ & $<0.001$ \\
\hline Birth weight $(\mathrm{kg})$ & $2.5(1.9-3.1)$ & $3.0(2.5-3.3)$ & $3.0(2.6-3.4)$ & $2.9(2.3-3.3)$ & $<0.001$ \\
\hline Small for gestational age $(\mathrm{n}(\%))$ & $28(22.6)$ & $415(14.8)$ & $801(8.6)$ & $43(6.7)$ & $<0.001$ \\
\hline Preeclampsia (n(\%)) & $42(33.9)$ & $338(12.1)$ & $353(3.8)$ & $16(2.5)$ & $<0.001$ \\
\hline
\end{tabular}

and diabetes were the most common in those with a gestational TA-eGFR range of $60-90 \mathrm{~mL} /$ $\min / 1.73 \mathrm{~m}^{2}$, and the prevalence decreased as the mothers' TA-eGFRs rose $(\mathrm{P}<0.001)$. This trend was similar for hypertension during pregnancy, systolic and diastolic blood pressures, and gestational diabetes. The baseline eGFR values were similar to those of gestational TAeGFR, and an increase of the median eGFR from baseline appeared in pregnancies in all subgroups, except for pregnancies with TA-eGFR of $60-90 \mathrm{~mL} / \mathrm{min} / 1.73 \mathrm{~m}^{2}$. The eGFR was most commonly measured once $(n=8,977,69.6 \%)$; otherwise, the $\mathrm{sCr} / \mathrm{eGFR}$ was measured twice or more in 2, $226(17.2 \%)$ and 1, $656(12.8 \%)$ cases, respectively.

\section{Maternal and fetal outcomes according to gestational eGFR ranges}

We investigated the association between TA-eGFR during gestation and adverse pregnancy outcomes, including preterm birth, low birth weight, SGA, preeclampsia, and these four features combined (Table 2). The lowest incidence of composite gestational complications was found in those with a TA-eGFR range of $120-150 \mathrm{~mL} / \mathrm{min} / 1.73 \mathrm{~m}^{2}$. Preeclampsia or SGA occurred most frequently in those with a TA-eGFR level lower than 90 $\mathrm{mL} / \mathrm{min} / 1.73 \mathrm{~m}^{2}$. This association was also replicated when we divided the study population by TA-eGFR range of $15 \mathrm{~mL} / \mathrm{min} / 1.73 \mathrm{~m}^{2}$ (Supplementary Table S1) (for all supplementary material see www.karger.com/doi/10.1159/000494746). Furthermore, when we plotted this association, a prominent non-linear, U-shaped association was shown between the TA-eGFR and composite pregnancy outcomes, as well as with preterm birth and low birth weight (Fig. 2). However, the risk of SGA or preeclampsia was inversely associated with gestational TA-eGFR. When we assessed the relationship between TA-eGFR and gestational complications in 10,937 pregnancies without underlying hypertension or diabetes mellitus, similar association was shown (Supplementary Fig. S1).

This association remained significant in the multivariable logistic regression analysis (Table 3). Moreover, when we divided our study population by TA-eGFR range of $15 \mathrm{~mL} /$ $\mathrm{min} / 1.73 \mathrm{~m}^{2}$, pregnancies with a TA-eGFR level of $120-135 \mathrm{~mL} / \mathrm{min} / 1.73 \mathrm{~m}^{2}$ had the lowest risk of preterm birth or low birth weight. Otherwise, the SGA and preeclampsia risks were increased in all pregnancy cases with lower TA-eGFR than in those with higher values (Supplementary Table S2).

Then, we analyzed eGFR levels in each trimester and considered the peak eGFR at each trimester as the representative level. When we analyzed 1,448 pregnancy cases with known first-trimester eGFR value, there was no significant association in the divided subgroups (Supplementary Table S3). However, the peak eGFR in the second trimester and that in the third trimester had almost the same association with our analysis with TA-eGFR values (Supplementary Tables S4 and S5). This unique association was not seen when subgroups were defined based on the available baseline eGFR ranges (Supplementary Table S6). 


\section{Kidney Blood Pressure Research}
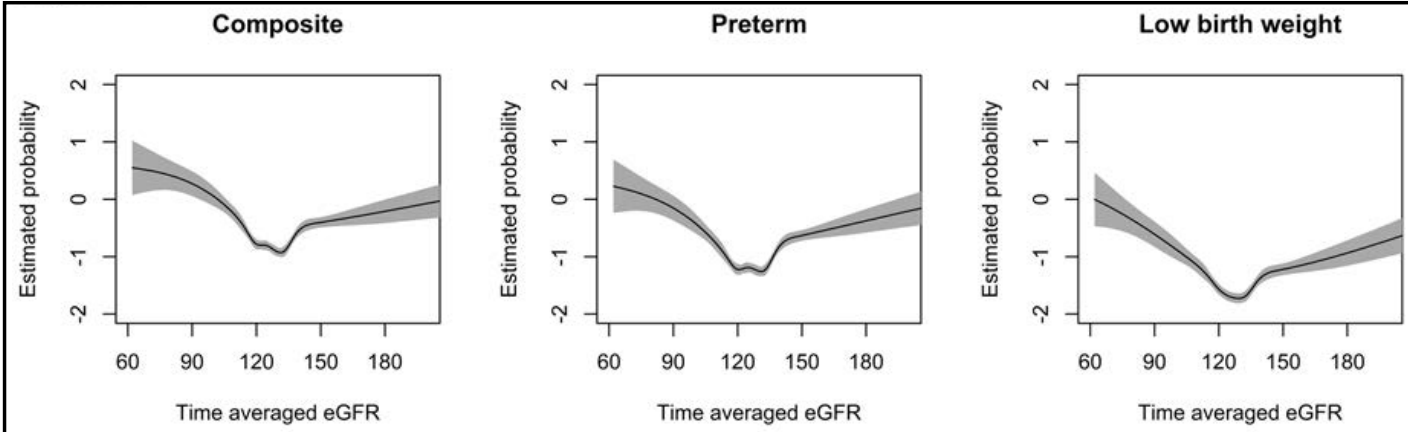

Small for gestational age

Preeclapmsia
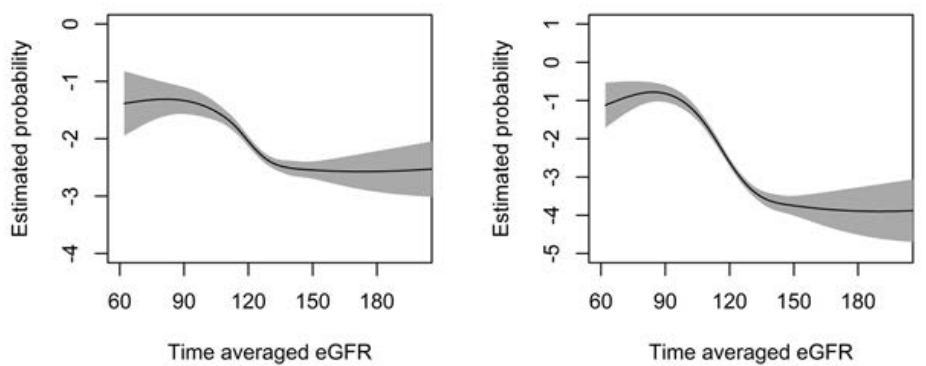

Fig. 2. Association between gestational eGFR and gestational complications. Generalized additive models demonstrating the relationship between the time-averaged gestational eGFR and the risk of adverse pregnancy outcomes, including (clockwise from the top left corner): composite, preterm, low birth weight, small for gestational age and preeclampsia. The dotted lines indicate the $95 \%$ confidence intervals of the estimated probability for each adverse pregnancy outcome. eGFR: estimated glomerular filtration rate.

Table 3. Risk of adverse pregnancy outcomes according to time-averaged eGFR during gestation. OR, Odds

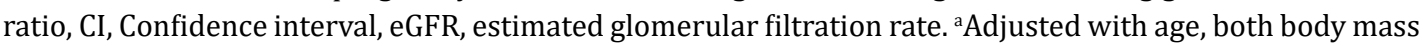
indexes at baseline, weight gain during pregnancy, multiparity, previous history of preterm birth, history of hypertension, diabetes mellitus, presence of hypertension during pregnancy, gestational diabetes mellitus. Missing value imputation by classification and regression trees method was performed to impute missing data in baseline body mass index (3146 cases), weight gain during gestation (2594 cases). ${ }^{\text {bHypertension }}$ during gestation was not adjusted in the multivariable model for preeclampsia risk, as preeclampsia itself was a hypertensive disorder

\begin{tabular}{|c|c|c|c|c|c|c|}
\hline \multirow{2}{*}{ Outcomes } & \multicolumn{3}{|c|}{ Univariable analysis } & \multicolumn{3}{|c|}{ aMultivariable analysis } \\
\hline & $\mathrm{OR}$ & $95 \% \mathrm{CI}$ & $P$ value & Adjusted OR & $95 \% \mathrm{CI}$ & P value \\
\hline \multicolumn{7}{|l|}{ Composite outcome } \\
\hline $\mathrm{eGFR} \geq 150 \mathrm{~mL} / \mathrm{min} / 1.73 \mathrm{~m}^{2}$ & 1.78 & $1.51-2.09$ & $<0.001$ & 1.64 & $1.38-1.95$ & $<0.001$ \\
\hline $120 \leq \mathrm{eGFR}<150 \mathrm{~mL} / \mathrm{min} / 1.73 \mathrm{~m}^{2}$ & Ref. & Ref. & & Ref. & Ref. & \\
\hline $90 \leq \mathrm{eGFR}<120 \mathrm{~mL} / \mathrm{min} / 1.73 \mathrm{~m}^{2}$ & 1.33 & $1.22-1.45$ & $<0.001$ & 1.41 & $1.28-1.56$ & $<0.001$ \\
\hline $60 \leq \mathrm{eGFR}<90 \mathrm{~mL} / \mathrm{min} / 1.73 \mathrm{~m}^{2}$ & 3.12 & 2.18-4.49 & $<0.001$ & 2.56 & $1.70-3.84$ & $<0.001$ \\
\hline \multicolumn{7}{|l|}{ Preterm birth } \\
\hline $\mathrm{eGFR} \geq 150 \mathrm{~mL} / \mathrm{min} / 1.73 \mathrm{~m}^{2}$ & 2.06 & $1.75-2.43$ & $<0.001$ & 1.96 & $1.65-2.34$ & $<0.001$ \\
\hline $120 \leq \mathrm{eGFR}<150 \mathrm{~mL} / \mathrm{min} / 1.73 \mathrm{~m}^{2}$ & Ref. & Ref, & & Ref. & Ref. & \\
\hline $90 \leq \mathrm{eGFR}<120 \mathrm{~mL} / \mathrm{min} / 1.73 \mathrm{~m}^{2}$ & 1.15 & $1.04-1.26$ & 0.005 & 1.16 & $1.04-1.30$ & 0.007 \\
\hline $60 \leq \mathrm{eGFR}<90 \mathrm{~mL} / \mathrm{min} / 1.73 \mathrm{~m}^{2}$ & 2.75 & $1.92-3.92$ & $<0.001$ & 2.29 & $1.53-3.43$ & $<0.001$ \\
\hline \multicolumn{7}{|l|}{ Low birth weight } \\
\hline $\mathrm{eGFR} \geq 150 \mathrm{~mL} / \mathrm{min} / 1.73 \mathrm{~m}^{2}$ & 1.84 & $1.53-2.21$ & $<0.001$ & 1.65 & $1.36-2.01$ & $<0.001$ \\
\hline $120 \leq \mathrm{eGFR}<150 \mathrm{~mL} / \mathrm{min} / 1.73 \mathrm{~m}^{2}$ & Ref. & Ref. & & Ref. & Ref. & \\
\hline $90 \leq \mathrm{eGFR}<120 \mathrm{~mL} / \mathrm{min} / 1.73 \mathrm{~m}^{2}$ & 1.38 & $1.24-1.53$ & $<0.001$ & 1.45 & $1.29-1.64$ & $<0.001$ \\
\hline $60 \leq \mathrm{eGFR}<90 \mathrm{~mL} / \mathrm{min} / 1.73 \mathrm{~m}^{2}$ & 3.35 & $2.32-4.80$ & $<0.001$ & 2.63 & $1.72-4.02$ & $<0.001$ \\
\hline \multicolumn{7}{|l|}{ Small for gestational age } \\
\hline $\mathrm{eGFR} \geq 150 \mathrm{~mL} / \mathrm{min} / 1.73 \mathrm{~m}^{2}$ & Ref. & Ref. & & Ref. & Ref. & \\
\hline $120 \leq \mathrm{eGFR}<150 \mathrm{~mL} / \mathrm{min} / 1.73 \mathrm{~m}^{2}$ & 1.30 & $0.96-1.81$ & 0.11 & 1.47 & $1.07-2.04$ & 0.02 \\
\hline $90 \leq \mathrm{eGFR}<120 \mathrm{~mL} / \mathrm{min} / 1.73 \mathrm{~m}^{2}$ & 2.42 & $1.76-3.39$ & $<0.001$ & 2.88 & $2.05-4.05$ & $<0.001$ \\
\hline $60 \leq \mathrm{eGFR}<90 \mathrm{~mL} / \mathrm{min} / 1.73 \mathrm{~m}^{2}$ & 4.04 & $2.38-6.79$ & $<0.001$ & 3.00 & $1.72-5.24$ & $<0.001$ \\
\hline \multicolumn{7}{|l|}{ bPreeclampsia } \\
\hline $\mathrm{eGFR} \geq 150 \mathrm{~mL} / \mathrm{min} / 1.73 \mathrm{~m}^{2}$ & Ref. & Ref. & & Ref. & Ref. & \\
\hline $120 \leq \mathrm{eGFR}<150 \mathrm{~mL} / \mathrm{min} / 1.73 \mathrm{~m}^{2}$ & 1.53 & $0.95-2.65$ & 0.10 & 1.54 & $0.91-2.61$ & 0.11 \\
\hline $90 \leq \mathrm{eGFR}<120 \mathrm{~mL} / \mathrm{min} / 1.73 \mathrm{~m}^{2}$ & 5.36 & $3.33-9.28$ & $<0.001$ & 5.68 & $3.31-9.76$ & $<0.001$ \\
\hline $60 \leq \mathrm{eGFR}<90 \mathrm{~mL} / \mathrm{min} / 1.73 \mathrm{~m}^{2}$ & 19.94 & $10.93-38.06$ & $<0.001$ & 11.42 & $5.77-22.62$ & $<0.001$ \\
\hline
\end{tabular}




\section{Kidney \\ Blood Pressure Research}
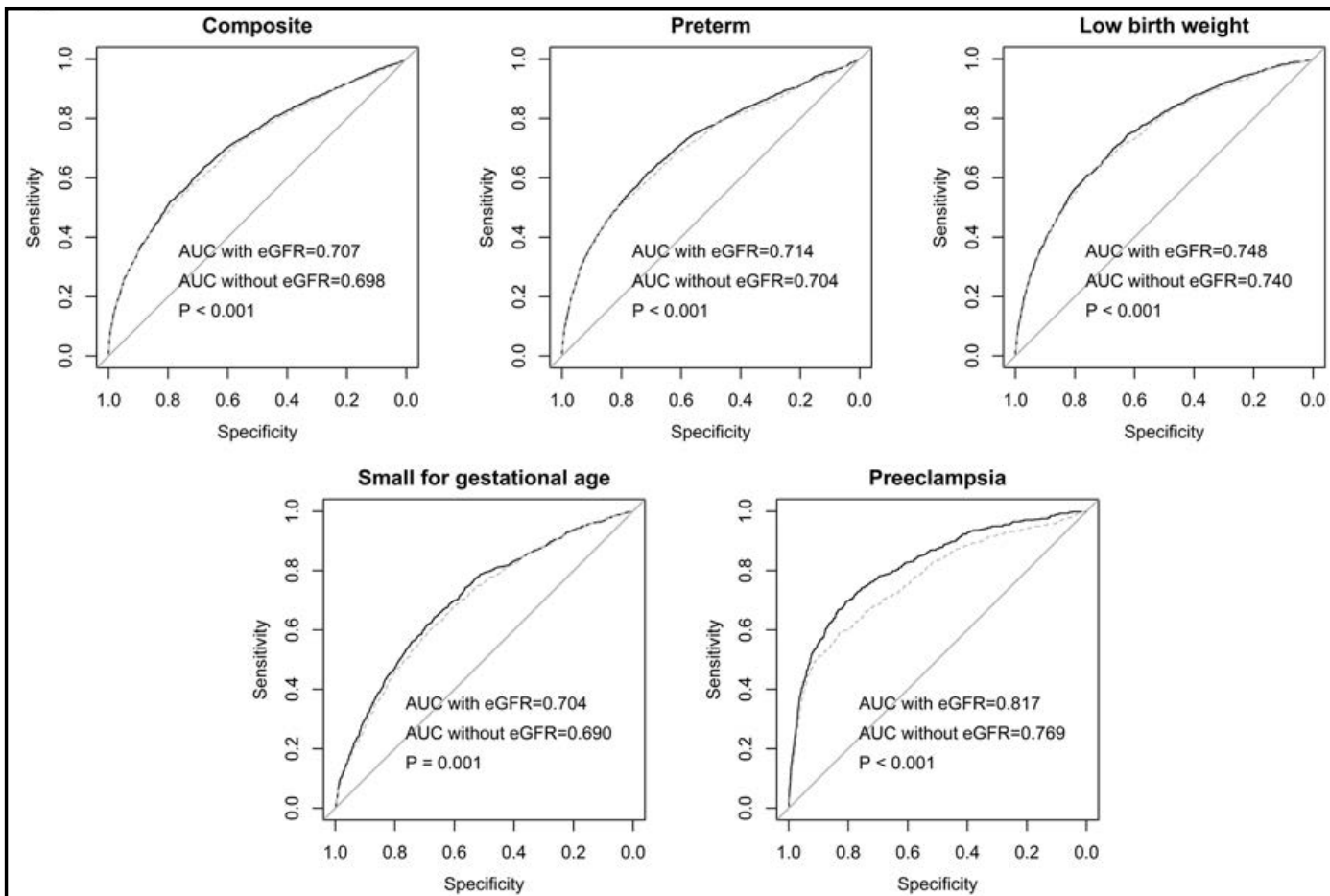

Fig. 3. Prediction model for gestational complications. ROC curve analysis was performed with multivariable models for each pregnancy outcome, with or without adding the risk scores that were defined by the eGFR ranges in the models. The black lines indicate the ROC curve of the model, including the eGFR ranges; and the dotted grey lines indicate the ROC curve without eGFR ranges. The x-axes show the specificity, and the $y$-axes show the specificity of the models. The calculated AUC and p-values for the difference in the AUC are also shown. eGFR: estimated glomerular filtration rate; AUC: area under the curve; ROC: receiver operating characteristic curve.

\section{Role of eGFR in predicting gestational complications}

We attempted to determine whether TA-eGFR information could enhance the predictability of the multivariable ROC curve model for gestational complications, and we constructed the models with and without adding the risk scores that were calculated using the TA-eGFR ranges (Fig, 3). Although the absolute increase of the AUC was small, adding the TA-eGFR risk scores to the model significantly increased its predictability for each adverse outcome.

\section{Change in eGFR and its association with pregnancy outcomes}

Underlying CKD was strongly associated with an increased risk of gestational complications when we investigated pregnancies of mothers with known eGFR and urinalysis results before gestation $(n=2,341)$ (Table 4). Furthermore, albuminuria was more commonly identified in those with baseline CKD than in those without.

Moreover, given the limited number of mothers with baseline renal function, we included all pregnancy cases of mothers with known baseline eGFR $(n=2,875)$. The median baseline eGFR was 120.1 (113.5-127.4) $\mathrm{mL} / \mathrm{min} / 1.73 \mathrm{~m}^{2}$, and we analyzed the relative eGFR change from baseline to the time-averaged gestation level (Table 5). The most frequent change in the CKD-EPI eGFR level was $5 \%$ to $15 \%$ from baseline $(n=1,099)$. Similarly, the incidence of preterm birth or low birth weight was low in pregnancies of women with mild eGFR elevation and increased in those with a higher eGFR elevation or eGFR decrease, although this result did not reach statistical significance in terms of preterm birth. The incidence of preeclampsia was higher in those with less eGFR increase, but the overall incidence of this outcome was small. 


\section{Kidney Blood Pressure Research}

Table 4. Clinical and gestational characteristics of mothers with available baseline renal function. $\mathrm{sCr}$, serum creatinine, eGFR, estimated glomerular filtration rate. Continuous variables are shown as median

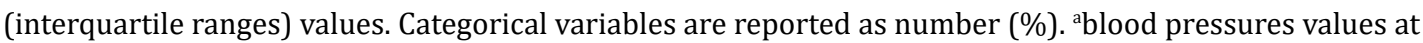
the time of admission for delivery. ${ }^{b}$ maximal value during gestational period. ${ }^{c}$ the values between 3 years before delivery and estimated conception date

\begin{tabular}{|c|c|c|c|}
\hline Characteristics & No evidence of CKD ( $\mathrm{n}=1980$ ) & Evidence of CKD stage 1 or $2(\mathrm{n}=361)$ & $P$ \\
\hline Age (years) & $33(31-36)$ & $33(31-35)$ & 0.76 \\
\hline Body mass index $\left(\mathrm{kg} / \mathrm{m}^{2}\right)$ & $21.0(19.4-23.0)$ & $21.7(20.1-24.5)$ & $<0.001$ \\
\hline Multiparity (n (\%)) & $1287(65.0)$ & $256(70.9)$ & 0.03 \\
\hline Presence of preterm birth history (n (\%)) & $234(11.8)$ & $45(12.5)$ & 0.79 \\
\hline Presence of stillbirth/miscarriage history (n (\%)) & 744 (37.6) & $136(37.7)$ & $>0.99$ \\
\hline Previous history of hypertension (n (\%)) & $261(13.2)$ & $108(29.9)$ & $<0.001$ \\
\hline Previous history of diabetes mellitus ( $\mathrm{n}(\%)$ ) & $178(9.0)$ & $29(8.0)$ & 0.63 \\
\hline Weight gain during pregnancy $(\mathrm{kg})$ & $12.8(10.0-16.0)$ & $12.1(8.5-15.1)$ & 0.005 \\
\hline Hypertension during pregnancy(n (\%)) & $91(4.6)$ & $52(14.4)$ & $<0.001$ \\
\hline aSystolic blood pressure ( $\mathrm{mmHg}$ ) & $120(110-128)$ & $124(116-136)$ & $<$ \\
\hline aDiastolic blood pressure (mmHg) & $72(6-80)$ & $76(68-86)$ & $<0.001$ \\
\hline Gestational diabetes mellitus (n (\%)) & $105(5.3)$ & $77(22.3)$ & $<0.001$ \\
\hline bUrine albumin (dipstick) (n (\%)) & & & $<0.001$ \\
\hline$(-)$ & $1604(85.7)$ & $221(63.9)$ & \\
\hline $1^{+}$ & $188(10.0)$ & $48(13.9)$ & \\
\hline $2^{+}$ & $50(2.7)$ & $39(11.3)$ & \\
\hline$>2^{+}$ & $29(1.5)$ & $28(8.1)$ & \\
\hline Cesarean section (n (\%)) & $1036(52.3)$ & $176(48.8)$ & 0.23 \\
\hline \multicolumn{4}{|l|}{ eGFR $\left(\mathrm{mL} / \mathrm{min} / 1.73 \mathrm{~m}^{2}\right)$} \\
\hline 'Baseline & $120.1(113.5-127.4)$ & $119.2(113.2-126.5)$ & 0.20 \\
\hline During gestation, time-averaged & $126.6(120.6-132.8)$ & $125.4(119.7-133.6)$ & 0.23 \\
\hline Composite outcome (n (\%)) & $444(22.4)$ & $110(30.5)$ & 0.007 \\
\hline Preterm birth & $293(14.8)$ & $81(22.4)$ & $<0.001$ \\
\hline Low birth weight & $215(10.9)$ & $52(14.4)$ & 0.06 \\
\hline Small for gestational age & $167(8.4)$ & $39(10.8)$ & 0.17 \\
\hline Preechmpsia & $61(3.1)$ & $33(9.1)$ & $<0.001$ \\
\hline
\end{tabular}

Table 5. Subgroups divided by eGFR change from baseline and their incidence of gestational complications

\begin{tabular}{|c|c|c|c|c|c|c|}
\hline Outcomes & $\begin{array}{c}\text { change }<-5 \% \\
(\mathrm{n}=413)\end{array}$ & $\begin{array}{c}-5 \% \leq \text { change }<+5 \% \\
(\mathrm{n}=845)\end{array}$ & $\begin{array}{c}+5 \% \leq \text { change }<+15 \% \\
(\mathrm{n}=1099)\end{array}$ & $\begin{array}{c}+15 \% \leq \text { change }<+25 \% \\
(\mathrm{n}=292)\end{array}$ & $\begin{array}{c}\text { change }>+25 \% \\
(\mathrm{n}=226)\end{array}$ & $P$ value \\
\hline Composite outcome & $102(24.7)$ & $208(24.6)$ & $243(22.1)$ & $74(25.3)$ & $61(27.0)$ & 0.43 \\
\hline Preterm birth (<37 weeks) (n (\%)) & $68(16.5)$ & $130(15.4)$ & $171(15.6)$ & $48(16.4)$ & $44(19.5)$ & 0.64 \\
\hline Moderate to late preterm $(32$ to $<37$ weeks) $(\mathrm{n}(\%))$ & $46(11.1)$ & $100(11.8)$ & $132(12.0)$ & $41(14.0)$ & $26(11.5)$ & 0.82 \\
\hline Very preterm $(28$ to $<32$ weeks) (n (\%)) & $10(2.4)$ & $13(1.5)$ & $21(1.9)$ & $6(2.1)$ & $6(2.7)$ & 0.77 \\
\hline Extremely preterm $(<28$ weeks) $(\mathrm{n}(\%))$ & $12(2.9)$ & $17(2.0)$ & $18(1.6)$ & $1(0.3)$ & $12(5.3)$ & 0.001 \\
\hline Gestational age (weeks) & $38(37-39)$ & $38(37-39)$ & $38(37-39)$ & $38(37-39)$ & $38(37-39)$ & 0.03 \\
\hline Low birth weight $(<2.5 \mathrm{~kg})(\mathrm{n}(\%))$ & $54(13.1)$ & $103(12.2)$ & $95(8.6)$ & $45(15.4)$ & $34(15.0)$ & 0.002 \\
\hline Very bw birth weight $(<1.5 \mathrm{~kg})(\mathrm{n}(\%))$ & $20(4.8)$ & $32(3.8)$ & $21(1.9)$ & $9(3.1)$ & $16(7.1)$ & 0.001 \\
\hline Birth weight $(\mathrm{kg})$ & $3.1(2.8-3.4)$ & $3.1(2.7-3.4)$ & $3.1(2.8-3.4)$ & $3.0(2.8-3.4)$ & $3.0(2.7-3.4)$ & 0.03 \\
\hline Small for gestational age (n (\%)) & $40(9.7)$ & $95(11.2)$ & $70(6.4)$ & $31(10.6)$ & $20(8.8)$ & 0.003 \\
\hline Preeclampsia (n (\%)) & $25(6.1)$ & $38(4.5)$ & $42(3.8)$ & $6(2.1)$ & $4(1.8)$ & 0.03 \\
\hline
\end{tabular}

\section{Discussion}

We found that the gestational eGFR levels were significantly associated with adverse pregnancy outcomes. A distinctive U-shaped relationship between eGFR and preterm birth or low birth weight was identified in a larger cohort than the one used in our previous study. The optimal gestational eGFR range in our study was $120-150$ or, specifically, 120-135 $\mathrm{mL} / \mathrm{min} / 1.73 \mathrm{~m}^{2}$. However, preeclampsia or SGA was much more common in those with lower eGFR values during pregnancy than in those with a high eGFR value. Consequently, we demonstrated that adding the gestational eGFR to a multivariable model improved its predictability.

The importance of appropriate hemodynamic adaptation during pregnancy for safe birth has been widely recognized [1]. Previously studied biomarkers that predicted worse pregnancy prognosis were also linked to this process. [3-6]. Mild renal impairment would cause inappropriate regulation of gestational adaptation to volume and vascular pressure change, resulting in obstetric complications. Therefore, renal function during gestation is important for predicting gestational outcome [7, 17]. However, the interpretation of 


\section{Kidney Blood Pressure Research}

Kidney Blood Press Res 2018;43:1688-1698

\begin{tabular}{l|l}
\hline DOI: 10.1159/000494746 & (c) 2018 The Author(s). Published by S. Karger AG, Base
\end{tabular}

Published online: 31 October 2018

www.karger.com/kbr

Park et al.: Pregnancy Complications and Gestational eGFR

commonly measured renal function parameters during pregnancy is challenging because laboratory indicators are altered due to gestational hyperfiltration, and the renal function is not assessed in many mothers before they become pregnant $[1,18]$. We previously reported the unique association between midterm eGFR and gestational outcomes, but that study only included mothers whose eGFR levels were measured during their second trimester [11]. The current study further suggests the clinical importance of gestational eGFR levels and provides evidence for appropriate interpretation of values. Furthermore, we also demonstrated that this association was replicated even in women without two well-known comorbidities related to renal hyperfiltration, diabetes mellitus, and hypertension.

The eGFR levels during pregnancy showed that there was a prominent U-shaped association with preterm birth and low birth weight. Some mothers with lower eGFR levels might have had reduced kidney function (e.g., $<60-90 \mathrm{~mL} / \mathrm{min} / 1.73 \mathrm{~m}^{2}$ ) before gestation because an increased eGFR occurs during pregnancy. Therefore, the baseline renal impairment and consequent inappropriate hemodynamic adaptation may have accounted for the increased risk of gestational complications.

For those with extremely high eGFR levels (e.g., $\geq 150 \mathrm{~mL} / \mathrm{min} / 1.73 \mathrm{~m}^{2}$ ), the high odds for developing preterm birth or low birth weight could be explained by several reasons. First, early CKD, in which an adaptive eGFR increase occurs, is a strong risk factor for worse pregnancy prognosis $[7,17]$. Some mothers with a gestational eGFR $\geq 150 \mathrm{~mL} / \mathrm{min} / 1.73 \mathrm{~m}^{2}$ might have early CKD with even more right-shifted eGFR levels and therefore experience more gestational complications. Second, over-hyperfiltration may indicate maladaptation to physiologic changes during pregnancy. Because the $\mathrm{sCr}$ levels are largely affected by volume, women with inappropriate hypervolemia would have high eGFR values. The function of the kidney may determine the hemodynamic reservoir during pregnancy, and extreme eGFR values may be related to the presence of a reduced baseline reservoir, leading to an inappropriately regulated volume state [19]. Lastly, because we used sCr-based eGFR levels, multiple factors that are related to the $\mathrm{sCr}$ levels should be considered [20, 21]. A supranormal eGFR or low $\mathrm{sCr}$ level could have been associated with poor dietary protein intake or low muscle mass, which may be also linked to worse gestational outcomes [20,21]. Although we attempted to control these confounding effect by adjusting the baseline BMI and weight change during pregnancy, our finding that low birth weight was common in those with higher eGFR values might be attributed to the idea that outcomes would be more prevalent in small mothers who would have lower $\mathrm{sCr}$ values.

For preeclampsia or SGA outcomes, the association between gestational eGFR and the odds for developing adverse events were different from those above because the risk of preeclampsia or SGA was significantly increased in those with lower eGFR levels than in those with higher levels. This may be because preeclampsia is defined by criteria that include increases in sCr value [22]. Therefore, many mothers with ongoing kidney injury that was confirmed by increases in sCr level would have been diagnosed with preeclampsia. In addition, when we accept the above hypothesis that high eGFR levels were associated with volume expansion, the preeclampsia risk may not be increased in the subgroup with low eGFR because the disease is mostly associated with depleted maternal volume [23, 24]. Moreover, as preterm birth was more common in mothers with higher eGFR values, they are less likely to have SGA outcomes, although the baby's birth weight was small because of their low gestational age.

The peak eGFR levels during the first trimester were not significantly associated with pregnancy outcomes. This period may be too early to assess the hemodynamic adaptation pattern that eGFR represents because gestational hyperfiltration develops in this trimester [1]. The clinical significance of the second-trimester eGFR suggests the possible usefulness of measuring renal function in mid-pregnancy, but the predictive value of an earlier assessment warrants further study.

The following limitations of our study should be considered. First, selection bias was not eliminated because mothers who had their kidney function measurement might have other 


\section{Kidney Blood Pressure Research}

ongoing medical reasons or complications for the examination. In addition, many mothers gave birth in our hospitals, which were tertiary referral centers, because of the presence of other risk factors (e.g. old age, coexisting comorbidities, or previous adverse pregnancy outcomes history). Additional studies should assess whether our result could be similarly applied to normal risk mothers, with a general screening measurement of renal function. Second, the CKD-EPI eGFR is not a gold-standard method to measure renal function during gestation. However, our method is beneficial compared with other widely used equations in population with an eGFR $\geq 60 \mathrm{~mL} / \mathrm{min} / 1.73 \mathrm{~m}^{2}$, and extreme overestimation with a low $\mathrm{sCr}$ level was less frequently shown with the CKD-EPI method [12, 25-27]. Moreover, besides its accuracy in reflecting the actual GFR, its value as an easily measured biomarker, significantly related to gestational outcomes, should be considered. Third, different definitions for gestational complications have been implemented among literatures, which should be considered when interpreting our study [28]. Still, although partial differences according to applied criteria may have existed, we believe the overall association will remain similar considering the general tendency of our study results. Fourth, we could not robustly study the significance of the eGFR changes from baseline through pregnancy because of limited availability. Additional studies with routine sCr/eGFR measurement before and after pregnancy may reveal the association between pregnancy outcomes and alterations of renal parameters. Lastly, the TA-eGFR was used to represent the eGFR level during gestation; however, the sCr/eGFR was measured only once in most mothers during the third trimester. A study with multiple eGFR measurements in a certain time interval may confirm our results.

\section{Conclusion}

Gestational eGFR had a unique association with obstetric complications. Appropriately interpreting gestational eGFR levels may help predict gestational complications in mothers. Furthermore, clinicians may consider kidney function measurement during pregnancy to assess ones' risk for adverse pregnancy outcomes. A future prospective study of normal risk mothers to test the predictive value of kidney function parameter is warranted.

\section{Disclosure Statement}

The authors of this manuscript state that they do not have any conflicts of interest and nothing to disclose.

\section{References}

1 Odutayo A, Hladunewich M: Obstetric nephrology: renal hemodynamic and metabolic physiology in normal pregnancy. Clin J Am Soc Nephrol 2012;7:2073-2780.

$\checkmark 2$ Davison JM, Dunlop W: Renal hemodynamics and tubular function normal human pregnancy. Kidney Int 1980;18:152-161.

-3 Smith GC, Crossley JA, Aitken DA, Jenkins N, Lyall F, Cameron AD, Connor JM, Dobbie R: Circulating angiogenic factors in early pregnancy and the risk of preeclampsia, intrauterine growth restriction, spontaneous preterm birth, and stillbirth. Obstet Gynecol 2007;109:1316-1324.

-4 Lim JH, Kim SY, Park SY, Yang JH, Kim MY, Ryu HM: Effective prediction of preeclampsia by a combined ratio of angiogenesis-related factors. Obstet Gynecol 2008;111:1403-1409.

-5 Zeisler H, Llurba E, Chantraine F, Vatish M, Staff AC, Sennstrom M, Olovsson M, Brennecke SP, Stepan H, Allegranza D, Dilba P, Schoedl M, Hund M, Verlohren S: Predictive Value of the sFlt-1:PlGF Ratio in Women with Suspected Preeclampsia. N Engl J Med 2016;374:13-22.

6 Polliotti BM, Fry AG, Saller DN, Mooney RA, Cox C, Miller RK: Second-trimester maternal serum placental growth factor and vascular endothelial growth factor for predicting severe, early-onset preeclampsia. Obstet Gynecol 2003;101:1266-1274. 


\section{Kidney \\ Blood Pressure Research}

Park et al.: Pregnancy Complications and Gestational eGFR

7 Piccoli GB, Cabiddu G, Attini R, Vigotti FN, Maxia S, Lepori N, Tuveri M, Massidda M, Marchi C, Mura S, Coscia A, Biolcati M, Gaglioti P, Nichelatti M, Pibiri L, Chessa G, Pani A, Todros T: Risk of Adverse Pregnancy Outcomes in Women with CKD. J Am Soc Nephrol 2015;26:2011-2022.

-8 Williams D, Davison J: Chronic kidney disease in pregnancy. BMJ 2008;336:211-215.

9 National Institute for Health and Care Excellence: Antenatal care for uncomplicated pregnancies. URL: https://www.nice.org.uk/guidance/cg62.

10 World Health Organization: WHO recommendations on antenatal care for a positive pregnancy experience. Geneva, 2016.

11 Park S, Lee SM, Park JS, Hong JS, Chin HJ, Na KY, Kim DK, Oh KH, Joo KW, Kim YS, Lee H: Midterm eGFR and Adverse Pregnancy Outcomes: The Clinical Significance of Gestational Hyperfiltration. Clin J Am Soc Nephrol 2017;12:1048-1056.

12 Levey AS, Stevens LA, Schmid CH, Zhang YL, Castro AF, 3rd, Feldman HI, Kusek JW, Eggers P, Van Lente F, Greene T, Coresh J: A new equation to estimate glomerular filtration rate. Ann Intern Med 2009;150:604612.

13 Jeong TD, Lee W, Yun YM, Chun S, Song J, Min WK: Development and validation of the Korean version of CKD-EPI equation to estimate glomerular filtration rate. Clin Biochem 2016;49:713-719.

14 Kidney Disease: Improving Global Outcomes (KDIGO) Work Group: KDIGO 2012 Clinical Practice Guideline for the evaluation and management of chronic kidney disease. Kidney Int Suppl 2013;3:136-150.

15 Howson CP, Kinney MV, McDougall L, Lawn JE: Born too soon: preterm birth matters. Reprod Health 2013;10:S1.

16 Blencowe H, Cousens S, Oestergaard MZ, Chou D, Moller AB, Narwal R, Adler A, Vera Garcia C, Rohde S, Say L, Lawn JE: National, regional, and worldwide estimates of preterm birth rates in the year 2010 with time trends since 1990 for selected countries: a systematic analysis and implications. Lancet 2012;379:21622172.

17 Piccoli GB, Attini R, Vasario E, Conijn A, Biolcati M, D’Amico F, Consiglio V, Bontempo S, Todros T: Pregnancy and chronic kidney disease: a challenge in all CKD stages. Clin J Am Soc Nephrol 2010;5:844-855.

18 Dunlop W: Serial changes in renal haemodynamics during normal human pregnancy. Br J Obstet Gynaecol 1981;88:1-9.

19 Piccoli GB, Attini R, Vigotti FN, Parisi S, Fassio F, Pagano A, Biolcati M, Giuffrida D, Rolfo A, Todros T: Is renal hyperfiltration protective in chronic kidney disease-stage 1 pregnancies? A step forward unravelling the mystery of the effect of stage 1 chronic kidney disease on pregnancy outcomes. Nephrology (Carlton) 2015;20:201-208.

20 Schummers L, Hutcheon JA, Bodnar LM, Lieberman E, Himes KP: Risk of adverse pregnancy outcomes by prepregnancy body mass index: a population-based study to inform prepregnancy weight loss counseling. Obstet Gynecol 2015;125:133-143.

21 Diemert A, Lezius S, Pagenkemper M, Hansen G, Drozdowska A, Hecher K, Arck P, Zyriax BC: Maternal nutrition, inadequate gestational weight gain and birth weight: results from a prospective birth cohort. BMC Pregnancy Childbirth 2016;16:224.

-22 American College of Obstetricians and Gynecologists' Task Force on Hypertension in Pregnancy: Hypertension in pregnancy. Executive Summary. Obstet Gynecol 2013;122:1122-1131.

23 Gallery ED, Hunyor SN, Gyory AZ: Plasma volume contraction: a significant factor in both pregnancyassociated hypertension (pre-eclampsia) and chronic hypertension in pregnancy. Q J Med 1979;48:593602.

24 Schrier RW, Briner VA: Peripheral arterial vasodilation hypothesis of sodium and water retention in pregnancy: implications for pathogenesis of preeclampsia-eclampsia. Obstet Gynecol 1991;77:632-639.

-25 Earley A, Miskulin D, Lamb EJ, Levey AS, Uhlig K: Estimating equations for glomerular filtration rate in the era of creatinine standardization: a systematic review. Ann Intern Med 2012;156:785-795.

-26 Levey AS, Bosch JP, Lewis JB, Greene T, Rogers N, Roth D: A more accurate method to estimate glomerular filtration rate from serum creatinine: a new prediction equation. Modification of Diet in Renal Disease Study Group. Ann Intern Med 1999;130:461-470.

27 Matsushita K, Tonelli M, Lloyd A, Levey AS, Coresh J, Hemmelgarn BR, Alberta Kidney Disease Network: Clinical risk implications of the CKD Epidemiology Collaboration (CKD-EPI) equation compared with the Modification of Diet in Renal Disease (MDRD) Study equation for estimated GFR. Am J Kidney Dis 2012;60:241-249.

28 Engle WA, Tomashek KM, Wallman C, Committee on Fetus and Newborn, American Academy of Pediatrics: "Late-Preterm" infants: a population at risk. Pediatrics 2007;120:1390-1401. 